\title{
A Note on the Convergence of Alternating Direction Methods
}

\author{
By Milton Lees
}

1. Introduction. Convergence of the single-parameter alternating direction methods of Douglas, Peaceman, and Rachford [1], [2], [3] has been proved for a wide class of elliptic difference equations; see, for example, Birkhoff and Varga [4]. The proof consists in showing that a certain matrix, similar to the defining matrix, has spectral radius less than one. Since the defining matrices for these methods are symmetric only when they are induced by a proper discretization of Laplace's equation in a rectangular region, an estimate for their spectral radii does not imply a corresponding norm estimate for their rate of convergence.

The purpose of this note is to present another proof of the convergence of the two basic alternating direction methods which, at the same time, provides a norm estimate for their rate of convergence. First, for simplicity, we shall consider Laplace's equation in an arbitrary, bounded lattice region. We shall prove that it is possible to select the acceleration parameter so that a suitable norm of the error is reduced after each iteration by the amount $1-c h+0\left(h^{2}\right)$, where $h$ is the uniform node spacing for the lattice, and $c>0$ depends only on the minimal eigenvalue for the Laplace operator. We shall indicate only briefly the extension of these results to elliptic equations with variable coefficients.

Our proof of the convergence of the alternating direction methods was motivated by two considerations; first, the close resemblance of the iteration equations to parabolic difference equations, and second, an integral estimate technique for proving that, under suitable conditions, the solutions of parabolic differential equations decay exponentially.

2. Formulation of the Problem. Let $\mathfrak{L}$ denote the (uniform) lattice of side $h$ determined by the nodes ( $\alpha h, \beta h), \alpha$ and $\beta$ being integers, positive, negative, or zero. Denote by $\bar{S}$ any finite subset of $\mathscr{L}$. In the usual way [5], we decompose $\bar{S}$ into two disjoint subsets: $S$, the interior nodes of $\bar{S}$, and $\partial S$, the boundary nodes of $\bar{S}$.

If $Q$ is any subset of $\mathscr{L}$, we denote by $\mathfrak{C}(Q)$ the linear space of all real-valued functions on $\mathscr{L}$ whose support is contained in $Q$. (In general, the support of a function $f$ is the closure of the set $\{p \mid f(p) \neq 0\}$.) Note that $\mathcal{C}(S)$ is finite-dimensional with dimension equal to the number of nodes in $S$. The advantage of thus trivially extending functions on $Q$ to all of $\mathscr{L}$ will become apparent later.

We denote by $\Delta$ the usual "five-point" Laplace difference operator, i.e.,

$$
\Delta u=u_{x \bar{x}}+u_{y \bar{y}}, u \in \mathfrak{C}(Q),
$$

where the subscripts $x$ and $y$ denote forward difference quotients, and $\bar{x}$ and $\bar{y}$ denote backward difference quotients [6].

Received July 20, 1961. The work presented in this paper was supported by the AEC Computing and Applied Mathematics Center, Institute of Mathematical Sciences, New York University. 
We shall be concerned, first, with the Dirichlet problem for the elliptic difference equation

$$
\Delta u=f \text { in } S,
$$

where $f \in \mathcal{C}(S)$. More precisely, we seek a function $u \in \mathfrak{C}(\bar{S})$ that satisfies (1.1) and assumes arbitrary prescribed values on $\partial S$.

It is easy to see that this problem is equivalent to the problem of solving a linear system of algebraic equations of order equal to the number of nodes in $S$. By means of the familiar maximum principle for the difference operator $\Delta$, one can show that this system of linear equations always has a unique solution. In this paper we shall be concerned with the two basic, single-parameter alternating direction methods for the inversion of this system of equations.

3. The Alternating Direction Methods. Let $w(0) \in \mathfrak{C}(\overline{\mathcal{S}})$ be a function that agrees with $u$ on $\partial S$, and otherwise arbitrary in $S ; w(0)$ will serve as the initial approximation to $u$. Now, for $n \geqq 1$, we determine two sequences of functions $\{w(n)\}$ and $\left\{w^{*}(n)\right\}$ in $\mathcal{e}(\bar{S})$ such that

$$
w(n)=w^{*}(n)=u \text { on } \partial S
$$

and

$$
\begin{aligned}
& \text { (a) } \rho^{-1}\left[w^{*}(n)-w(n-1)\right]=w_{x \dot{x}}^{*}(n)+w_{y \bar{y}}(n-1)-f \\
& \text { (b) } \rho^{-1}\left[w(n)-w^{*}(n)\right]=w_{y \dot{y}}(n)-w_{y \bar{y}}(n-1)
\end{aligned}
$$

in $S$. Here $\rho>0$, the so-called acceleration parameter, is to be selected later. These equations define what is usually referred to as the Douglas-Rachford alternating direction method for solving $\Delta u=f$.

In [3] it is shown that (2.1) and (2.2) determine uniquely (alternately) the functions $w^{*}(n)$ and $w(n)$ in terms of $w(n-1)$ and the values of $u$ on $\partial S$. This operation taking $w(n-1)$ into $w(n)$ involves only the inversion of approximately $2 \sqrt{p}$ tridiagonal matrices, where $p$ is the number of nodes in $S$.

Following Douglas and Rachford, we eliminate from (2.2) the auxiliary function $w^{*}(n)$. Adding (2.2a) to (2.2b), we obtain the equation

$$
D w(n)=w_{x \tilde{x}}^{*}(n)+w_{y \tilde{y}}(n)-f,
$$

where we have introduced the notation

$$
D w(n)=\rho^{-1}[w(n)-w(n-1)] .
$$

Solving (2.2b) for $w^{*}$, we get

$$
w^{*}(n)=w(n)-\rho^{2} D w_{y \bar{y}}(n) .
$$

Hence,

$$
w_{x \bar{x}}^{*}(n)=w_{x \bar{x}}(n)-\rho^{2} \Lambda D w(n),
$$

where $\Lambda$ is defined as follows:

$$
\Lambda u=u_{x \bar{x} y \bar{y}} .
$$


Putting the value just obtained for $w_{x \dot{x}}^{*}(n)$ into (2.3), we find that $w$ is a solution of the fifth-order difference equation

$$
D w=\Delta w-\rho^{2} \Lambda D w-f, \text { in } S, \text { for } n \geqq 1 .
$$

The other alternating direction method, the Peaceman-Rachford method, is very similar to this; it is characterized by the equations

$$
\rho^{-1}\left[w^{*}(n)-w(n-1)\right]=w_{x \dot{x}}^{*}(n)+w_{y \bar{y}}(n-1)-f
$$

and

$$
\rho^{-1}\left[w(n)-w^{*}(n)\right]=w_{x \bar{x}}^{*}(n)+w_{y \bar{y}}(n)-f .
$$

After eliminating from these equations the auxiliary function $w^{*}(n)$, we find that $w$ is a solution of the fifth-order difference equation

$$
D w=2 \Delta w-\rho \Delta D w-\rho^{2} \Lambda D w-2 f \text { in } S, \text { for } n \geqq 1 \text {. }
$$

Our problem, now, is to show that $w(n) \rightarrow u$, as $n \rightarrow \infty$.

4. Preparation. We define on $\mathrm{e}(S)$ an inner product $\langle u, v\rangle$ as follows:

$$
\langle u, v\rangle=h^{2} \sum_{P \in \mathcal{L}} u(P) v(P) .
$$

The norm $\langle v, v\rangle^{1 / 2}$ induced by this inner product will be denoted by $\|v\|$. We require two additional norms for $\mathfrak{e}(S)$ :

and

$$
\|v\|_{1}=\left(\left\|v_{\bar{x}}\right\|^{2}+\left\|v_{\bar{y}}\right\|^{2}\right)^{1 / 2}
$$

$$
\|v\|_{2}=\left(\|v\|^{2}+\rho^{2}\left\|v_{\bar{x} \dot{y}}\right\|^{2}\right)^{1 / 2} \text {. }
$$

A subscript $S$ will be placed on these quantities whenever we want to indicate that the sum is to be extended only over $S$.

In [7] it is proved that the minimal eigenvalue $\lambda$ of the Laplace difference operator $\Delta$, relative to $S$, can be characterized as follows:

$$
\lambda=\inf _{0 \neq v \in e(s)} \frac{\|v\|_{1, s}^{2}}{\|v\|^{2}} .
$$

Since $\|v\|_{1, s} \leqq\|v\|_{1}$, it follows immediately that

$$
\lambda\|v\|^{2} \leqq\|v\|_{1}^{2},
$$

for all $v \in \mathbb{C}(S)$.

On the strength of this inequality, we now prove the

LEMma 1. Any function $v \in \mathcal{C}(S)$ satisfies the inequality

$$
\|v\|_{1}^{2} \geqq \lambda_{0}\|v\|_{2}^{2}
$$

where

$$
\lambda_{0}=\frac{\lambda}{1+2 \lambda \rho^{2} h^{-2}} .
$$

Proof. We have from (3.1) and the definition of $\|v\|_{2}$ that

$$
\|v\|_{1}^{2}-\lambda\|v\|_{2}^{2} \geqq-\lambda \rho^{2}\left\|v_{\bar{x} \bar{v}}\right\|^{2} \text {. }
$$


To this inequality we apply the elementary inequality [8]

$$
h^{2}\left\|v_{\dot{x} \bar{y}}\right\|^{2} \leqq 2\|v\|_{1}^{2},
$$

valid for all $v \in \mathcal{C}(S)$, and we find that

$$
\|v\|_{1}^{2}-\lambda\|v\|_{2}^{2} \geqq-2 \lambda \rho^{2} h^{-2}\|v\|_{1}^{2},
$$

which is equivalent to (3.2).

5. Convergence of the Alternating Direction Methods. Let $u \in \mathfrak{C}(\overline{\mathcal{S}})$ be the solution of the Dirichlet problem for $\Delta u=f$ in $S$, and let $w(n)$ be the corresponding solution of the Douglas-Rachford equation (2.4). Then, by linearity, the error function $v(n)=u-w(n)$ belongs to the linear space $\mathcal{C}(S)$, and satisfies; for $n \geqq 1$, the difference equation

$$
P v \equiv D v-\Delta v+\rho^{2} \Lambda D v=0
$$

in $S$. Let $\phi(n)=(1+\rho \mu)^{-n}$, where $\mu>0$. One verifies immediately that $\phi$ is a solution of the difference equation

$$
D \phi=-\mu \phi \text {. }
$$

Theorem 1. If $\mu \leqq \lambda_{0}$, then the error function $v(n)$ for the Douglas-Rachford method satisfies the inequalities

$$
\|v(n)\|_{i} \leqq \phi(n)\|v(0)\|_{i} \quad(i=1,2)
$$

Proof. We set

$$
M=2 \rho \sum_{\xi=1}^{n} \phi^{-2}(\xi)\langle v(\xi), P v(\xi)\rangle
$$

Since $v(\xi) \in \mathbb{C}(S)$, we see that $M=0$.

Introduce the function $z(\xi) \in \mathcal{C}(S)$ by means of the formula

$$
v(\xi)=\phi(\xi) z(\xi) .
$$

Then we have that

$$
D v=\phi D z+z D_{\phi}-\rho D \phi \cdot D z
$$

which, in view of (4.2) becomes

$$
D v=\phi[a D z-\mu z],
$$

where $a=\phi^{-1}(1)>0$.

From this and (4.1) it follows that

$$
P v=\phi\left[a D z-\mu z-\Delta z+a \rho^{2} \Lambda D z-\rho^{2} \mu \Lambda z\right]=\phi[a D z+B z],
$$

where $B$ is defined in the obvious way.

Because of (4.5), (4.3) becomes

$$
M=\rho \sum_{\xi=1}^{n} 2\langle z(\xi), a D z(\xi)+B z(\xi)\rangle .
$$


We now recall from [8] the following quadratic difference identities

$$
\begin{gathered}
2\langle z, D z\rangle=D\|z\|^{2}+\rho\|D z\|^{2}, \\
2\langle z, \Delta D z\rangle=D\left\|z_{x \bar{y}}\right\|^{2}+\rho\left\|D z_{x \bar{j}}\right\|^{2}, \\
\langle z, \Delta z\rangle=-\|z\|_{1}^{2},
\end{gathered}
$$

and

$$
\langle z, \Lambda \bar{z}\rangle=\left\|z_{\dot{x} \bar{y}}\right\|^{2} .
$$

Using these identities in (4.6), we obtain, after some slight rearrangement, the identity

$$
M=\rho \sum_{\xi=1}^{n}\left\{a D\|z\|_{2}^{2}+2\left(\|z\|_{1}^{2}-\mu\|z\|_{2}^{2}\right)+a^{2}\|D z\|_{2}^{2}+\mu a\|D z\|_{1}^{2}\right\} .
$$

Since $\|z\|_{1}^{2} \geqq \lambda_{0}\|z\|_{2}^{2}$, by Lemma 1 , and $\mu \leqq \lambda_{0}$, we have from (4.11) that

$$
M \geqq \rho \sum_{\xi=1}^{n} a D\|z\|_{2}^{2}=a\|z(n)\|_{2}^{2}-a\|z(0)\|_{2}^{2} .
$$

But, since $M=0$, we obtain from this and (4.4) that

$$
\|v(n)\|_{2}=\phi(n)\|z(n)\|_{2} \leqq \phi(n)\|z(0)\|_{2}=\phi(n)\|v(0)\|_{2},
$$

which proves the second inequality of the theorem.

Next, we set

$$
N=\rho \sum_{\xi=1}^{n} \phi^{-2}(\xi)\|P v(\xi)\|_{s}^{2} .
$$

Then, by (4.4) and (4.5), (4.12) becomes

But

$$
N=\rho \sum_{\xi=1}^{n}\|a D z(\xi)+B z(\xi)\|_{s}^{2} .
$$

$$
\|a D z+B z\|_{s}^{2}=a^{2}\|D z\|_{s}^{2}+\|B z\|_{s}{ }^{2}+2 a\langle D z, B z\rangle_{s} \geqq a^{2}\|D z\|^{2}+2 a\langle D z, B z\rangle,
$$
where we have dropped the subscript $S$, because $D z \in \mathcal{C}(S)$. Hence,

$$
N \geqq \rho \sum_{\xi=1}^{n}\left\{a^{2}\|D z\|^{2}+2 a\langle D z, B z\rangle\right\} .
$$

Using the identities (4.7)-(4.10) again, we obtain from (4.13) the inequality

$$
N \geqq \rho \sum_{\xi=1}^{n}\left\{a^{2}\|D z\|^{2}+a D\|z\|_{1}^{2}-\mu a D\|z\|_{2}^{2}+a \rho\|D z\|_{1}^{2}\right\} .
$$

Therefore, by adding a combination of the above inequalities,

$$
N+\mu M \geqq \rho a \sum_{\xi=1}^{n} D\|z\|_{1}^{2}
$$

Again, $N+\mu M=0$, so that

$$
\|z(n)\|_{1} \leqq\|z(0)\|_{1},
$$

which, in view of (4.4), implies that

$$
\|v(n)\|_{1} \leqq \phi(n)\|v(0)\|_{1} .
$$

This completes the proof of the theorem. 
Since $\rho \mu>0$, Theorem 1 implies that the Douglas-Rachford method is convergent, but we also have the stronger result:

ThEOREM 2. If $\mu=\lambda_{0}$ and $\rho=h / \sqrt{2 \lambda}$, then the error function $v(n)$ for the DouglasRachford alternating direction method satisfies the inequalities

$$
\|v(n)\|_{i} \leqq\left(1+\frac{\sqrt{2 \lambda}}{4} h\right)^{-n}\|v(0)\|_{i} \quad(i=1,2) .
$$

Proof. The inequality (4.14) follows from Theorem 1, by minimizing $\phi(n)$ as a function of $\rho$.

In an analogous fashion, we can prove

Theorem 3. If $\mu=2 \lambda_{0}\left(1-\lambda_{0} \rho\right)^{-1}$ and $\rho=h / \sqrt{2 \lambda}$, then the error function $v(n)$ for the Peaceman-Rachford alternating direction method satisfies the inequality

$$
\|v(n)\|_{1} \leqq\left(\frac{4-\sqrt{2 \lambda} h}{4+\sqrt{2 \lambda} h}\right)^{n}\|v(0)\|_{1} .
$$

Note that the Peaceman-Rachford method has a smaller convergence factor.

The preceding results can be extended to more general elliptic difference equations, e.g., to those that come from elliptic differential equations of the form

$$
\frac{\partial}{\partial x}\left(a(x, y) \frac{\partial u}{\partial x}\right)+\frac{\partial}{\partial y}\left(b(x, y) \frac{\partial u}{\partial y}\right)=f(x, y)
$$

in a bounded, open set $\Omega$. The functions $a$ and $b$ must belong to $c^{1}(\bar{\Omega})$. The main point that we wish to emphasize is that, because the inner product $\langle u, v\rangle$ is defined as a sum over all of $\mathscr{L}$, one must be able to extend $a$ and $b$ in a continuously differentiable way to a slightly larger set $\omega \supset \bar{\Omega}$. This can be carried out, for instance, by means of the Whitney extension theorem [9], in a form given by Hörmander [10].

One can also show that similar results can be proved for the Douglas-Rachford method as applied to elliptic equations in $n$ independent variables, although this is not the case with the Peaceman-Rachford method [8].

New York University

New York 3, New York

1. J. Douglas, JR., "A note on the alternating direction implicit method," Proc. Amer Math. Soc., v. 8, 1957, p. 409-411.

2. D. W. PEACEMAN \& H. H. RAChFord, JR., "The numerical solution of parabolic and elliptic differential equations," $J$. Soc. Indust. Appl. Math., v. 3, 1955, p. 28-41.

3. J. Douglas, JR. \& H. H. RACHFORD, JR., "On the numerical solution of heat conduction problems in two and three space variables," Trans. Amer. Math. Soc., v. 82, 1956, p. 421-439.

4. G. BIRKHOFF \& R. S. VARGA, "Implicit alternating direction methods," Trans. Amer. Math. Soc., v. 92, 1959, p. 13-24.

5. L. BERs, "On mildly nonlinear partial difference equations of elliptic type," J. Res. Nat. Bur. Standards, v. 51, 1953, p. 229-236.

6. M. LEES, " $A$ priori estimates for the solutions of difference approximations to parabolic differential equations," Duke Math. J., v. 27, 1960, p. 297-312.

7. R. Courant, K. O. Friedrichs, \& H. Lewy, "Über die Partiellen Differenzengleichungen der Mathematischen Physik," Math. Ann., v. 100, 1928, p. 32-74.

8. M. LEEs, Alternating Direction and'Semi-Explicit Difference Methods for Parabolic Partial Differential Equations, N.Y.U. Tech. Report, Institute of Mathematical Sciences,

9. H. Whitney, "Analytic extensions of differentiable functions defined in closed sets," Trans. Amer. Math. Soc., v. 36, 1934, p. 63-89.

10. L. HörMANDER, "On the division of distributions by polynomials," Ark. Mat., v. 3, 1954 , p. 555-568. 